\title{
The "rotation-activity connection": Its extension to photospheric activity diagnostics
}

\author{
S. Messina ${ }^{1}$, M. Rodonò ${ }^{1,2}$, and E. F. Guinan ${ }^{3}$ \\ 1 Catania Astrophysical Observatory, Via S. Sofia 78, 95125 Catania, Italy \\ e-mail: sme@sunct.ct.astro.it \\ 2 Dept. of Physics and Astronomy, University of Catania, Via S. Sofia 78, 95125 Catania, Italy \\ e-mail: mrodono@alpha4.ct.astro.it \\ 3 Dept. of Astronomy and Astrophysics, Villanova University, Villanova 19085, PA, USA \\ e-mail: edward.guinan@villanova.edu
}

Received 19 May 1999 / Accepted 25 October 2000

\begin{abstract}
In this paper we present the results of a different approach in the study of the so-called rotation-activity connection, which is a well established correlation between rotation and magnetic activity at chromospheric and outer atmospheric levels. The present study concerns the photospheric level and was carried out by using $V$-band photometric light curve amplitudes as indicators of starspot coverage and of magnetic activity. A high degree of correlation between the envelope of maximum $V$-band light curve amplitudes and the rotation period is found for the active star members of young open clusters (IC 2602, IC 2391, Alpha Persei, Pleiades and Hyades), as well as for active field stars. This correlation shows a different behaviour in two different rotation period ranges. Moreover, some evidence of a possible activity "saturation" is found among the most rapidly rotating stars of the sample. Additional correlations between photospheric and other magnetic activity indicators in the chromosphere, transition region and corona are also investigated. The results presented here can be considered as an extension of the well established rotation-activity connection valid from the corona, transition region and chromosphere, down to the photosphere.
\end{abstract}

Key words. stars: activity - late-type - magnetic fields - rotation - starspots

\section{Introduction}

The cool starspots that appear on the photospheres of stars characterised by high angular momentum and convective envelopes $(\mathrm{F}-\mathrm{M})$ are thought to be produced, as on the Sun, by the emergence into the photosphere of bundles of magnetic flux tubes generated in the stellar interior by magnetodynamic processes. The dynamo mechanism is believed to originate near the base of the convective envelope (Parker 1979; Schüssler 1983). Since starspots are the sites of strong magnetic fields, the total percent area covered by spots can provide an indirect measure of the magnetic filling factor $\left(f_{\mathrm{S}}\right)$ by spots. The filling factor $\left(f_{\mathrm{S}}\right)$ is the fraction of the photosphere covered by magnetic fields confined to spots, and a measure of the total magnetic flux density in spots $\left(f_{\mathrm{S}} B\right)$, if the magnetic field intensity $(B)$ is known. Other important sites of magnetic fields on the stellar photosphere are bright plages and network elements of total percent area $f_{\mathrm{P}}$. The covering factor of spots and plages $\left(f_{\mathrm{T}}=f_{\mathrm{S}}+f_{\mathrm{P}}\right)$ represents a direct measure of the total magnetic filling factor. In contrast to starspots and bright plages, other magnetic activity indicators, such as chromospheric and TR's emission line fluxes, can actually provide only an indirect estimate of the magnetic field (Pallavicini 1992).

Because the value of $f_{\mathrm{T}}$ is related to the total magnetic energy that the dynamo action can provide, it appears to be a suitable parameter for investigating the dependence of the dynamo action on global stellar parameters. Estimates of $f_{\mathrm{S}}$ can be obtained from photometric data by light curve inversion methods based on recently developed "spot models" (Rodonò et al. 1995; Lanza et al. 1998). Non-polarimetric methods, based on the comparison of the broadening excess of Zeeman sensitive with respect to Zeeman insensitive lines (e.g., Mathys 1989; Saar 1990, 1996), are quite successful in determining the properties (total flux density and $f_{\mathrm{P}}$ ) of bright magnetic photospheric regions, but are not applicable to dark photospheric regions. Moreover, the spot contribution to the line profile is negligible at optical wavelengths, compared with the contribution of bright magnetic regions. 
In this paper we propose to estimate the magnetic filling factor $\left(f_{\mathrm{S}}\right)$ from photometric data. We accomplish this by using a parameter which is closely related to the total fractional area covered by starspots: the maximum peak-to-peak amplitude of the optical-band flux rotational modulation. These newly determined values of $f_{\mathrm{S}}$ for a sample of single active late-type stars are used to study the dependence of the fraction of photospheric magnetic activity, confined to dark spots, on global stellar parameters and, in particular, to investigate the rotation-activity connection at the photospheric level.

\section{Maximum light curve amplitude as an activity indicator}

Since photospheric spots are the sites of strong magnetic fields, the convective transport of heat is strongly inhibited and this is the reason why the spots appear darker than the surrounding photosphere. Therefore, cool spots can produce the observed optical flux modulation as they travel across the visible hemisphere and disappear behind the limb of a rotating star.

The amplitude of the modulation depends on several geometrical and physical parameters. Some parameters are constant in time, such as the inclination of the star's rotation axis; or can be assumed to be constant, such as the brightness contrast between spot and photosphere; others are variable, such as the total area covered by spots and their surface distribution. The photometric precision of presently available observations generally does not allow us to distinguish between single huge stellar spots or compact groups of small solar-like spots. Let us briefly summarise the effects of these various parameters on the light modulation amplitude:

- inclination of the stellar rotation axis and latitude of the spot: the modulation amplitude depends on both the inclination $(i)$ of the star's rotation axis and the latitude where the spots or spot groups are centred, as can be inferred from the following two extreme cases: a) "pole-on" star $\left(i=0^{\circ}\right)$ : any spot is equally visible at all rotation phases and, therefore, spots can not produce any rotational modulation of the observed flux;

b) "equator-on" $\operatorname{star}\left(i=90^{\circ}\right)$ : except polar caps, spots are carried by the rotation from a maximum visibility, at the disk centre line, to a minimum visibility, when they disappear from view; while approaching the stellar limb, the limb-darkening effect further depresses the spot's brightness contrast with respect to the photosphere; hence, equatorial spots on equator-on stars produce the highest amplitude modulation;

- temperature and colour indices: the amplitude of the modulation depends also on the ratio between spot and photospheric brightness or temperature.
This ratio is likely to be constant in time and time sequences of light curves of several stars have been successfully modelled (e.g., Rodonò et al. 1995; Lanza et al. 1998; Messina et al. 1999a, 1999b) without any need of changing this ratio. Nevertheless, there exists some indication that this ratio may be variable for different types of stars (Byrne et al. 1995; O'Neal et al. 1998). In the case of the Sun, the spot temperature is inversely correlated to the spot area (Kopp $\&$ Rabin 1992). The reddening of the colour indices at light minima, i.e. when the maximum fraction of the projected stellar disk is covered by spots, suggests that, although the spot temperatures are lower than the photospheric temperature, their temperature is several hundreds of degrees $K$, otherwise the spots would not be able to produce any variation of the colour indices;

- spot areas and longitudes: the amplitude of the modulation depends on both the total fractional area covered by spots and on the asymmetry of their longitudinal distribution; the absence of flat maxima in most of the observed light curves and the long-term variation of the brightness maximum suggest that the fraction of the photosphere covered by spots $\left(f_{\mathrm{S}}\right)$ can be considered as the sum of two terms: $f_{\mathrm{u}}$ and $f_{\text {as }}$,

$f_{\mathrm{S}}=f_{\mathrm{u}}+f_{\mathrm{as}} \leq 1$

where $f_{\mathrm{u}}$ represents the minimum areal fraction of spots which are always visible and, therefore, unable to produce any flux modulation, though the star's brightness appears somewhat depressed. In equator-on stars $\left(i=90^{\circ}\right)$, we expect bands of spots evenly distributed in longitude and/or polar caps to contribute to $f_{\mathrm{u}}$. In non equator-on stars $\left(i<90^{\circ}\right)$ we may expect an additional contribution from polar spots. At any given epoch, $f_{\mathrm{u}}$ determines the difference $\left(\Delta V_{\mathrm{u}}\right)$ between the presumed "unspotted" (brightest) magnitude and the "spotted" magnitude at the light curve maximum. Hereafter, the $V$-band will be considered as an illustrative example.

The parameter $f_{\text {as }}$ represents the fraction of spots which are asymmetrically distributed in longitude and are responsible of the flux modulation. Their distribution and total area determine the light curve shape and mean magnitude, respectively. Thus $f_{\text {as }}$ determines the peak-to-peak light curve amplitude $\left(\Delta V_{\text {as }}\right)$. Therefore, the total brightness variation $(\Delta V)$ from the presumed unspotted level

$\Delta V=\Delta V_{\mathrm{u}}+\Delta V_{\text {as }}$

may be used to estimate the total spotted area (uniformly + asymmetrically distributed in longitude components) at any given epoch.

However, it must be pointed out that for active stars, such as those analysed in this paper, a completely unspotted level will possibly never become observable. In fact, even the much less active Sun shows a few 


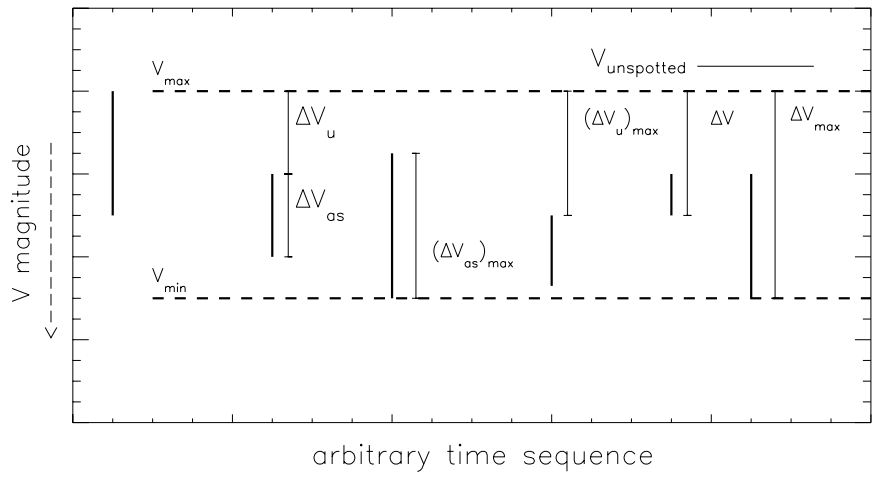

Fig. 1. An illustrative sample of light curve amplitudes (heavy solid bars) is represented to show the meaning of the symbols used in the text: (from left to right) $V_{\max }$ and $V_{\min }$ are the star's brightest and faintest magnitudes ever observed; $\Delta V_{\text {as }}$ is the observed light curve amplitude and $\Delta V_{\mathrm{u}}$ is the difference between the brightest magnitude and the light curve maximum at a given epoch; $\left(\Delta V_{\mathrm{as}}\right)_{\max }$ and $\left(\Delta V_{\mathrm{u}}\right)_{\max }$ are their maximum historic values, respectively; $\Delta V$ is the difference between $V_{\max }$ and the light curve minimum at a given epoch; $\Delta V_{\max }$ is the maximum value of the difference between $V_{\max }$ and the faintest magnitude $\left(V_{\text {min }}\right)$ ever observed; $V_{\text {unspotted }}$ is the magnitude corresponding to a completely unspotted visible hemisphere, that may, but does not necessarily, coincide with $V_{\max }$

spots at its activity minimum. Hence, the brightest observed magnitude $\left(V_{\max }\right)$ may differ (see Fig. 1) from the true unspotted magnitude (Neff et al. 1995; O'Neal et al. 1996) and $\Delta V_{\mathrm{u}}$ and $\Delta V$ must be considered as lower limits of the uniformly distributed $\left(f_{\mathrm{u}}\right)$ and of the total fraction $\left(f_{\mathrm{S}}\right)$ of spotted area, respectively, at any given epoch. Only from a large number of light curves covering a sufficiently extended time interval the value of $V_{\max }$ can reliably approach $V_{\text {unspotted }}$.

Since bright plages may compensate at some level the light dimming effects of dark spots, the value of $\Delta V_{\text {as }}$ must be considered as a lower limit of the asymmetrically distributed spotted area. However, for the most active stars at least, the effect of starspots dominates (Radick et al. 1989, 1998) and generally plages are not required to obtain quite satisfactory model light curves (Messina et al. 1999a, 1999b; Lanza et al. 1998; Rodonò et al. 1995), so that plage contamination was disregarded in the present study.

On the basis of these considerations $\Delta V=\Delta V_{\mathrm{u}}+V_{\text {as }}$ represents a lower limit on the total spot covering fraction $\left(f_{\mathrm{S}}\right)$, that approaches the true value if an extended long term study is available.

We believe that the amplitude of the long-term variation $\left(\Delta V_{\max }=V_{\max }-V_{\min }\right)$, the maximum values of $\Delta V_{\mathrm{u}}$ and $\Delta V_{\text {as }}\left[\left(\Delta V_{\mathrm{u}}\right)_{\max }\right.$ and $\left(\Delta V_{\text {as }}\right)_{\max }$, respectively $]$ obtained from an extended time series of light curves represent the best estimates (though certainly lower limits) of the maximum spottedness $\left(f_{\max }\right)$ and of the extreme values of the uniform $\left(f_{\mathrm{u}}\right)$ and asymmetric $\left(f_{\text {as }}\right)$ components of the spotted area on a given star. A graphic illustration

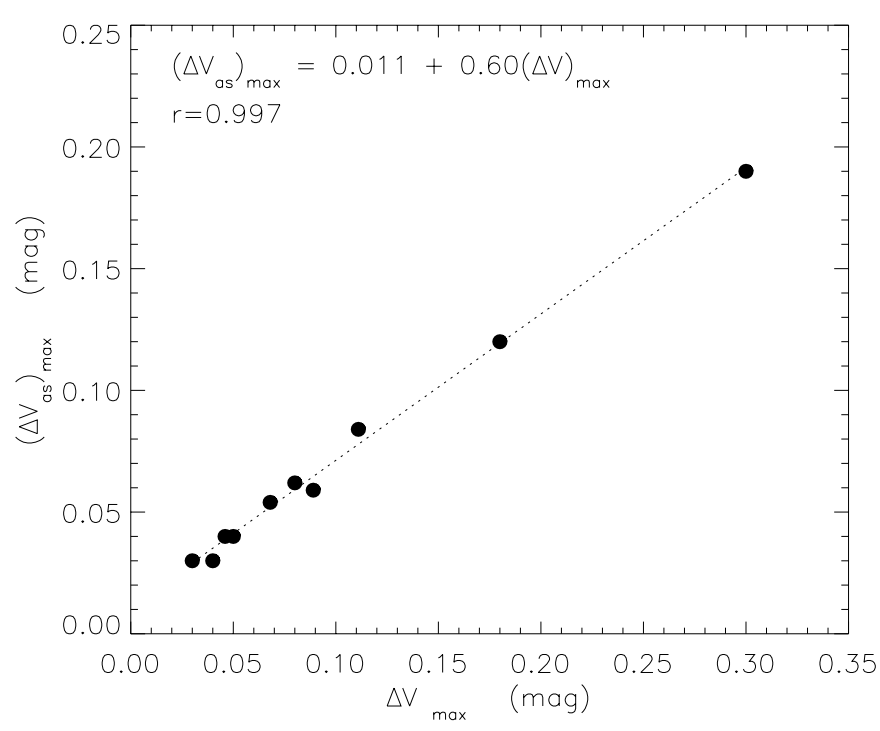

Fig. 2. $\left(\Delta V_{\text {as }}\right)_{\max }$ vs. $\Delta V_{\max }$ for a sample of extensively observed single active stars (Table 8 ). The dotted line is the fitting linear relation

of the parameters we use in this section is presented in Fig. 1 .

By analysing a sample of single late-type stars with rather extended time series of light curves (see Table 8), it has been noted (Messina 1998) that $\left(\Delta V_{\text {as }}\right)_{\max }$ increases with increasing total spotted area $\left(\Delta V_{\max }\right)$. The existence, at least for extensively observed single stars, of such a strong correlation between $\left(\Delta V_{\text {as }}\right)_{\max }$ and $\Delta V_{\max }$, as shown in Fig. 2, indicates that not only $\Delta V_{\max }$, but also the light curve maximum amplitude $\left(\Delta V_{\text {as }}\right)_{\max }$ from time series of light curves can be adopted as suitable indicator of a lower limit of the star maximum spottedness $\left(f_{\max }\right)$.

The last result is very important if we consider that the value of $\Delta V_{\max }$, which most closely approaches $V_{\text {unspotted }}$, has been measured for a small sample of stars, whereas the value of $\left(\Delta V_{\text {as }}\right)_{\max }$, which does not depend on $V_{\text {unspotted }}$, is obviously available for a much larger sample of stars.

\section{Analysis and discussion}

For the present study we selected from the literature a sample of 225 active single stars according to the following criteria: a) single main-sequence stars, because it is not clear, yet, how both binarity and evolution of the internal structure outside of the main sequence influence the magnetic activity; b) stars belonging to stellar clusters of known age (IC 2391, IC 2602, Alpha Persei, Pleiades and Hyades clusters) in order to study age effects on the magnetic activity, as well field stars; c) stars with known rotation periods; d) stars with well determined light curve amplitudes (in the $V$-band of the Johnson's $U B V$ system) (Tables 3-8).

In an earlier study by O'Dell et al. (1995) the dependence of the light curve amplitude on the rotation period and on the inverse Rossby number $\left(R\left(\equiv R_{0}{ }^{-1}\right)=\tau_{\mathrm{c}} / P\right.$, where $\tau_{\mathrm{c}}$ is the convective turnover time at the base of the 

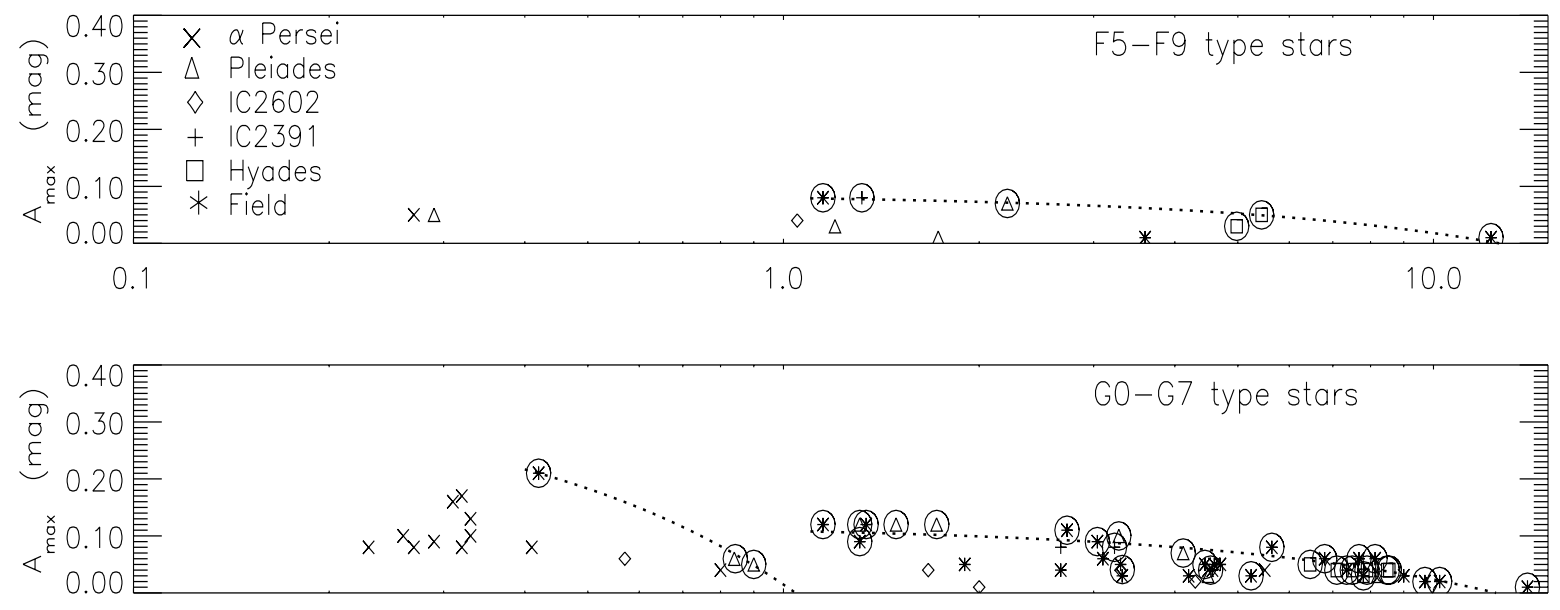

0.1

1.0

10.0

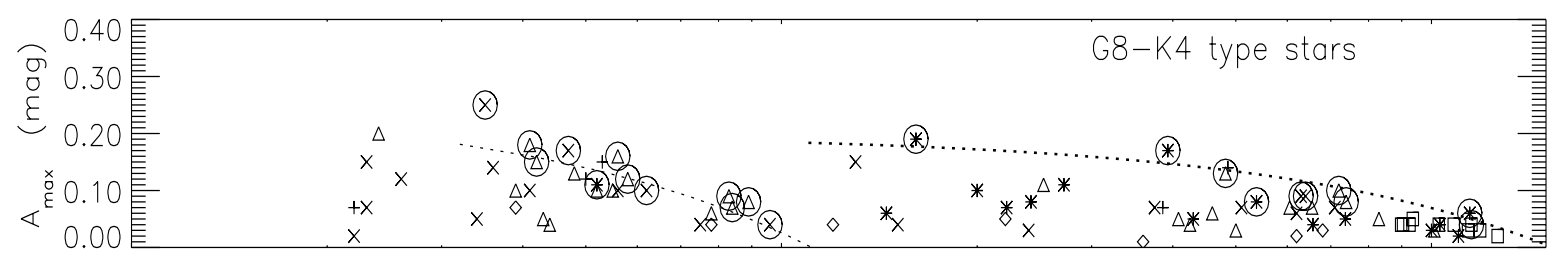

0.1

1.0

10.0

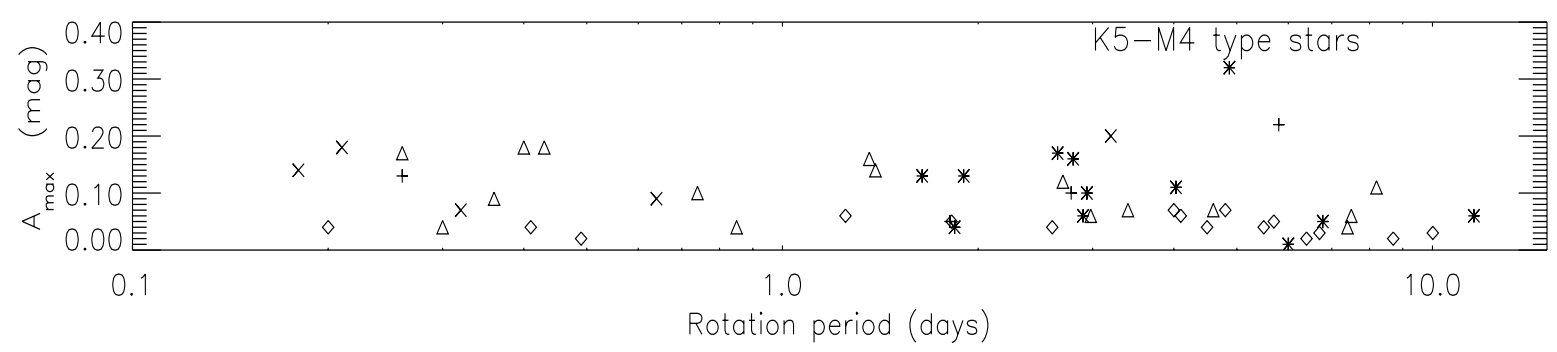

Fig. 3. $A_{\max }$ versus rotation period $(P)$. The data upper envelopes (circled symbols) both in the ultra fast and fast rotation ranges are best fitted (dotted line) by a linear law for F, G and K-type stars. Note that the abscissa is logarithmic

Table 1. The Rossby number $\left(R_{0}\right)$ ranges in which different slopes can be identified, as shown in Fig. 4

\begin{tabular}{ccc}
\hline type & ultra fast & fast \\
\hline $\mathrm{F}$ & & $R_{0}>0.18$ \\
$\mathrm{G}$ & $R_{0} \leq 0.10$ & $R_{0}>0.10$ \\
$\mathrm{~K}$ & $R_{0} \leq 0.06$ & $R_{0}>0.06$ \\
\hline
\end{tabular}

convection zone) was suggested on qualitative grounds. The existence of such a dependence was later questioned by Krishnamurthi et al. (1998) on the basis of their newly determined rotation periods for a sample of Pleiades cluster stars.

Using a sample of stars much larger than that considered by O'Dell et al. and Krishnamurthi et al., we investigate the existence of correlations between $\left(\Delta V_{\text {as }}\right)_{\max }$ (hereafter referred to as $A_{\max }$ ), the parameter we have chosen as indicator of photospheric magnetic activity confined into spots, and global stellar parameters, such as
$B-V$ colour index, rotation rate and other activity indicators.

\subsection{Activity-rotation relation}

Apart from the field stars, whose interstellar reddening can be neglected because of their proximity to the Sun, all the stars in our sample were first dereddened using the mean $B-V$ colour excess values as used by Pinsonneault et al. (1998). Then, in order to minimise the colour effects (i.e., mass for main sequence stars and convective zone depth) on the activity-rotation relation, we divided our sample into four colour ranges:

$$
\begin{aligned}
& +0.50 \leq B-V \leq+0.57 \quad(\mathrm{~F} 5-\mathrm{F} 9) \\
& +0.58 \leq B-V \leq+0.73 \quad(\mathrm{G} 0-\mathrm{G} 7) \\
& +0.74 \leq B-V \leq+1.13 \quad(\mathrm{G} 8-\mathrm{K} 4) \\
& +1.14 \leq B-V \leq+1.70 \quad(\mathrm{~K} 5-\mathrm{M} 4)
\end{aligned}
$$

Each star was assigned to one of the four spectral ranges only on the basis of the $B-V$ colour index. 

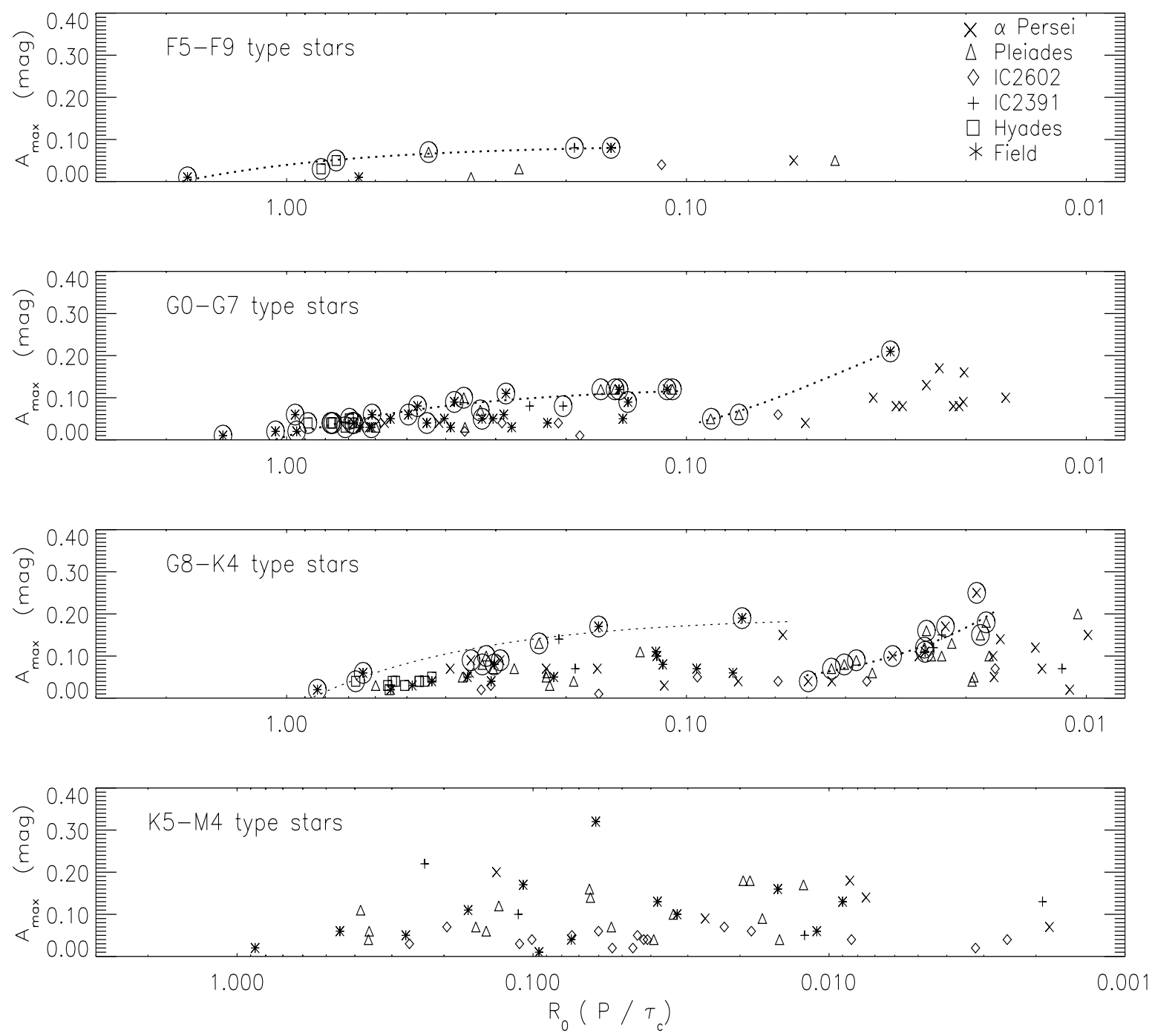

Fig. 4. $A_{\max }$ versus Rossby number $\left(R_{0}\right)$. The data upper envelopes (circled symbols) are fitted (dotted line) in the fast rotation range by a linear law for $\mathrm{F}, \mathrm{G}$ and $\mathrm{K}$-type stars. The $A_{\max }-R_{0}$ relation in the ultra ultra fast rotation range is best fitted by a power law for $\mathrm{G}$ and K-type stars. Note that the abscissa is logarithmic

Figure 3 shows that the upper envelope of $A_{\max }$ is a decreasing function of the rotation period $(P)$, as expected from the dynamo theory that predicts the magnetic flux density to increase with rotation velocities. However, different quantitative behaviours of the upper envelope of $A_{\text {max }}$ versus $P$ are apparent in the following different rotation rate ranges:
$P \leq 1.10$ (d) (hereafter called ultra fast rotators)
$P>1.10$ (d) (hereafter called fast rotators).

The different behaviour in these rotation ranges is best seen among K-type stars (see also Fig. 5), which are the most numerous stars in our sample. We can argue that a similar behaviour may be present in the other mass regimes, but the currently available data are too sparse to support this behaviour as strongly as in the G8-K4 regime. The scatter of $A_{\max }$ at any given rotation period can be due to:

a) the dependence of the magnetic activity on colour, as discussed later on;

b) the different inclination of the rotation axis with respect to the line of sight, as explained in Sect. 2;

c) the paucity of the available light curves for most of the stars in our sample;

d) the different degree of long-term symmetry/asymmetry in the spot distribution.

It can be noted in the ultra fast regime that the $A_{\max }$ upper envelopes of the $\mathrm{G}$ and K-type stars (the most numerous in our sample) have their maximum values at about $P \simeq 0.35(\mathrm{~d})$ and they do not exceed such values even among the faster rotators $(P \lesssim 0.35 \mathrm{~d})$. A similar behaviour is more clearly apparent when the Rossby 
Table 2. Correlation coefficients $(r)$, number of stars used to define the upper envelope $(N)$, parameters of the linear, exponential and power law weighted fits to the upper data envelope of the $A_{\max }-P$ and $A_{\max }-R_{0}$ relations and reduced chi-squares $\left(\chi_{\nu}^{2}\right)$

\begin{tabular}{|c|c|c|c|c|c|c|c|c|c|c|}
\hline Sp. Type & $r$ & $N$ & $a$ & $b$ & $\chi_{\nu}^{2}$ & $\log _{10} a$ & $\chi_{\nu}^{2}$ & $\log _{10} a$ & $b$ & $\chi_{\mathrm{r}}^{2}$ \\
\hline ultra fast & otation & & \multicolumn{3}{|c|}{$A_{\max }=a+b P$} & \multicolumn{2}{|c|}{$\log _{10} A_{\max }=\log _{10} a+b P$} & \multicolumn{3}{|c|}{$\log _{10} A_{\max }=\log _{10} a+b \log _{10} P$} \\
\hline G0-G7 & -0.99 & 3 & $0.23 \pm 0.46$ & $-0.33 \pm 0.88$ & 2.56 & $-0.58 \pm 0.46-1.29 \pm 0.88$ & 2.76 & $-0.52 \pm 0.45$ & $-1.88 \pm 1.31$ & 2.88 \\
\hline G8-K4 & -0.89 & 12 & $0.25 \pm 0.50$ & $-0.23 \pm 0.88$ & 0.29 & $-0.45 \pm 0.50-0.83 \pm 0.88$ & 0.21 & $-1.23 \pm 0.35$ & $-1.20 \pm 1.25$ & 0.17 \\
\hline \multicolumn{11}{|c|}{ fast rotation } \\
\hline F5-F9 & -0.94 & 6 & $0.08 \pm 0.49$ & $-0.007 \pm 0.09$ & 0.15 & $-1.05 \pm 0.50-0.08 \pm 0.09$ & 0.20 & $-0.98 \pm 0.54$ & $-0.75 \pm 0.99$ & 0.24 \\
\hline G0-G7 & -0.91 & 29 & $0.13 \pm 0.06$ & $-0.010 \pm 0.01$ & 0.30 & $-0.88 \pm 0.06-0.05 \pm 0.01$ & 0.31 & $-0.75 \pm 0.07$ & $-0.58 \pm 0.10$ & 0.40 \\
\hline G8-K4 & -0.86 & 10 & $0.20 \pm 0.04$ & $-0.013 \pm 0.01$ & 0.50 & $-0.68 \pm 0.05-0.05 \pm 0.01$ & 0.50 & $-0.60 \pm 0.05$ & $-0.60 \pm 0.07$ & 0.60 \\
\hline
\end{tabular}

$$
A_{\max }=a+b R_{0} \quad \log _{10} A_{\max }=\log _{10} a+b R_{0} \quad \log _{10} A_{\max }=\log _{10} a+b \log _{10} R_{0}
$$

ultra fast rotation

\begin{tabular}{ll|l|lll|lll|lll} 
G0-G7 & -0.90 & 3 & $0.21 \pm 0.37$ & $-2.04 \pm 8.41$ & 2.05 & $-0.58 \pm 0.37-7.91 \pm 8.41$ & 1.13 & $-0.46 \pm 1.55$ & $-1.14 \pm 1.08$ & 1.81 \\
G8-K4 & -0.86 & 12 & $0.25 \pm 0.49$ & $-4.76 \pm 17.97$ & 0.46 & $-0.45 \pm 0.48-17.04 \pm 15.97$ & $0 . .30$ & $-2.86 \pm 1.86$ & $-1.23 \pm 1.16$ & 0.26 \\
\hline
\end{tabular}

fast rotation

\begin{tabular}{ll|l|lll|lll|lll} 
F5-F9 & -0.92 & 6 & $0.08 \pm 0.49$ & $-0.05 \pm 0.67$ & 0.16 & $-1.06 \pm 0.49-0.58 \pm 0.67$ & 0.20 & $-1.03 \pm 0.52$ & $-0.75 \pm 0.95$ & 0.23 \\
G0-G7 & -0.86 & 29 & $0.11 \pm 0.06$ & $-0.09 \pm 0.12$ & 0.25 & $-0.91 \pm 0.06-0.53 \pm 0.12$ & 0.25 & $-0.74 \pm 0.05$ & $-0.60 \pm 0.12$ & 0.33 \\
G8-K4 & -0.89 & 10 & $0.19 \pm 0.04$ & $-0.22 \pm 0.11$ & 0.43 & $-0.69 \pm 0.04-0.86 \pm 0.11$ & 0.37 & $-1.33 \pm 0.05$ & $-0.53 \pm 0.06$ & 0.31 \\
\hline
\end{tabular}

number is used (Fig. 4). The activity-rotation relations are derived by considering the data upper envelopes, which most closely approach the true maximum light curve amplitude at any given rotation period. The stars we used to compute the $A_{\max }$ upper envelope fits are marked by the spectral type ("--fit") in the last column of Tables 3 to 8 and are represented by circled symbols in the Figs. 3 to 5 . The upper envelope of $A_{\max }$ appears to be strongly correlated with both the rotation period and the Rossby number (Table 1 and Fig. 4), with different decreasing slopes in passing from ultra fast to fast rotators.

The Rossby number $R_{0}$ was computed adopting the empirical turnover time given by Noyes et al. (1984) for the $+0.50 \leq B-V \leq+1.36$ colour range (F-K type) and the theoretical turnover time from the Girardi et al. (2000) models for the $+1.37 \leq B-V \leq+1.70$ colour range (M-type).

We argue that the boundary between ultra fast and fast stars, which is clearest in the G8-K4 regime, falls at the same rotation period for other masses (Fig. 3). Therefore, when $A_{\max }$ is plotted versus Rossby number $\left(R_{0}\right)$ the breaking boundaries would be masked (Fig. 4) by the spurious colour dependence introduced in the normalisation of the rotation period $(P)$ by means of a colour dependent function $\left(\tau_{\mathrm{c}}\right)$. For this reason the different spectral type ranges are plotted separately in Fig. 4.

Though all the data are lower limits, the discontinuity shown by the upper envelope of $A_{\max }$ in passing from ultra fast to fast stars (Fig. 5) is unlikely to be an artifact. It is clearly apparent in the $\mathrm{K}$ colour range and, even if much less clearly, in the $\mathrm{G}$ range. For K-type stars, the discontinuity amplitude $(0.20 \mathrm{mag})$ is almost 20 times larger than

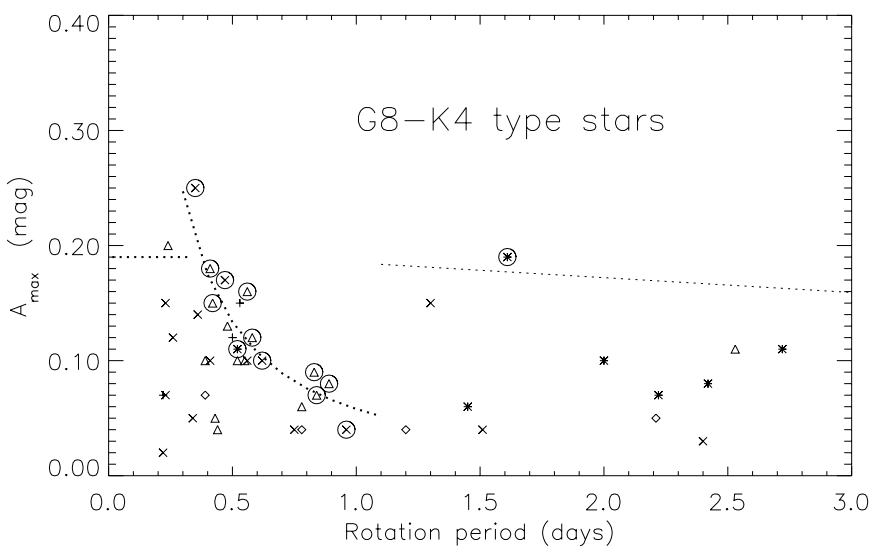

Fig. 5. $A_{\max }$ versus rotation period $(P)$ in the rotation range 0-3 days blown up from Fig. 3 for G8-K4 stars. The symbols have same meaning than in Figs. 3, 4

the standard deviation of the residuals with respect to the data fit for ultra fast stars (dotted line in Figs. 4 and 5). For the F stars too few ultra fast rotators are present in our sample to confidently support a similar behaviour.

Because of the detection of this discontinuity in the $A_{\text {max }}-P$ relation, a monitoring program of ultra fast stars was started. Additional photometry of 21 stars in the Pleiades and Alpha Persei clusters (Messina 2000) confirms that the upper envelope of the maximum $V$-band light curve amplitudes increases with rotation velocity, within the rotation range $0.4-1.1$ days.

The data upper envelopes were fitted by linear, exponential and power law relations. The $A_{\max }-P$ relation (both in the ultra fast and fast rotation ranges) is best 
Table 3. Pleiades cluster active stars. The stars that were considered for the $A_{\max }-P$ upper envelope fits are identified in the last column

\begin{tabular}{|c|c|c|c|c|c|c|c|c|c|}
\hline Star & $\begin{array}{l}\text { Other } \\
\text { name }\end{array}$ & $(B-V)_{0}$ & $\begin{array}{l}P_{\text {rot }} \\
\text { (d) }\end{array}$ & $\begin{array}{l}A_{\max } \\
(\mathrm{mag})\end{array}$ & $\begin{array}{l}v \sin i \\
\left(\mathrm{~km} \mathrm{~s}^{-1}\right)\end{array}$ & $R_{0}$ & Ref. & $\begin{array}{c}\# \\
\text { of curves }\end{array}$ & Notes \\
\hline $\begin{array}{l}\text { HCG20 } \\
\text { HCG71 }\end{array}$ & & $\begin{array}{l}1.34 \\
1.34\end{array}$ & $\begin{array}{l}2.70 \\
2.98\end{array}$ & $\begin{array}{l}0.15 \\
0.04\end{array}$ & - & $\begin{array}{l}0.130 \\
0.144\end{array}$ & $\begin{array}{l}9 \\
9\end{array}$ & $\begin{array}{l}1 \\
1\end{array}$ & \\
\hline $\begin{array}{l}\text { HII34 } \\
\text { HII133 }\end{array}$ & $\begin{array}{l}\text { V810 Tau } \\
\text { V623 Tau }\end{array}$ & $\begin{array}{l}0.88 \\
1.27\end{array}$ & $\begin{array}{l}6.553 \\
1.36\end{array}$ & $\begin{array}{l}0.07 \\
0.16\end{array}$ & $\begin{array}{l}7.3^{(a)} \\
9.0\end{array}$ & $\begin{array}{l}0.304 \\
0.064\end{array}$ & $\begin{array}{l}7 \\
9\end{array}$ & $\begin{array}{l}1 \\
1\end{array}$ & \\
\hline $\begin{array}{l}\text { HII152 } \\
\text { HII191 }\end{array}$ & V936 Tau & $\begin{array}{l}0.64 \\
1.27\end{array}$ & $\begin{array}{l}4.12 \\
3.1\end{array}$ & $\begin{array}{l}0.07 \\
0.04\end{array}$ & $11.1^{(a)}$ & $\begin{array}{l}0.328 \\
0.145\end{array}$ & $\begin{array}{l}4 \\
9\end{array}$ & $\begin{array}{l}1 \\
1\end{array}$ & $G_{--} f i t$ \\
\hline HII250 & & 0.64 & 0.843 & 0.06 & $6.9^{(a)}$ & 0.074 & 8 & 1 & $G_{--} f i t$ \\
\hline $\begin{array}{l}\text { HII253 } \\
\text { HII263 }\end{array}$ & & $\begin{array}{l}0.64 \\
0.84\end{array}$ & $\begin{array}{l}1.721 \\
4.82\end{array}$ & $\begin{array}{l}0.12 \\
0.17\end{array}$ & $\begin{array}{l}38.2^{(a)} \\
10\end{array}$ & $\begin{array}{l}0.151 \\
0.234\end{array}$ & $\begin{array}{l}8 \\
9\end{array}$ & $\begin{array}{l}1 \\
1\end{array}$ & $\begin{array}{l}G_{-} f i t \\
K_{-f i t}\end{array}$ \\
\hline HII293 & & 0.66 & 4.5: & 0.03 & $6.6^{(a)}$ & 0.358 & 6 & 1 & \\
\hline HII296 & V966 Tau & 0.82 & 2.53 & 0.11 & $14.7^{(a)}$ & 0.131 & 4 & 1 & \\
\hline HII314 & V1038 Tau & 0.62 & 1.49 & 0.12 & $38.0^{(a)}$ & 0.164 & 8 & 2 & $G_{--} f i t$ \\
\hline HII320 & & 0.84 & 4.6: & 0.06 & 110.8 & 0.223 & 6 & 1 & \\
\hline HII324 & V632 Tau & 1.00 & 0.41 & 0.18 & 73.0 & 0.018 & 5 & 1 & $K_{-} f i t$ \\
\hline HII335 & MX Tau & 1.22 & 0.36 & 0.09 & 73.0 & 0.017 & 5 & 1 & \\
\hline HII345 & & 0.80 & 0.84 & 0.065 & $18.9^{(a)}$ & 0.043 & 8 & 2 & $K_{-} f i t$ \\
\hline HII357 & NP Tau & 1.17 & 3.4: & 0.07 & 7.5 & 0.156 & 6 & 1 & \\
\hline HII625 & V811 Tau & 1.15 & 0.428 & 0.18 & 94.0 & 0.019 & 5 & 1 & \\
\hline HII686 & OU Tau & 1.23 & 0.396 & 0.18 & 64.0 & 0.018 & 7 & 2 & \\
\hline HII708 & V1085 Tau & 0.58 & 0.294 & 0.05 & 46.0 & 0.042 & 8 & 2 & \\
\hline HII 727 & V855 Tau & 0.51 & 1.2 & 0.04 & 50.0 & 0.262 & 3 & 1 & \\
\hline HII738 & V1041 Tau & 1.13 & 0.83 & 0.09 & 50.0 & 0.038 & 9 & 2 & $K_{-f i t}$ \\
\hline HII739 & V969 Tau & 0.62 & 0.90 & 0.05 & $14.0^{(a)}$ & 0.087 & 8 & 2 & $G_{--} f i t$ \\
\hline HII879 & V813 Tau & 1.03 & 7.387 & 0.08 & $7.2^{(a)}$ & 0.324 & 7 & 1 & $K_{-f i t}$ \\
\hline HII 882 & & 1.03 & 0.58 & 0.12 & 65.0 & 0.025 & 7 & 1 & $K_{-f i t}$ \\
\hline HII883 & V789 Tau & 1.04 & 7.2 & 0.10 & $6:$ & 0.317 & 9 & 1 & $K_{-f i t}$ \\
\hline HII885 & & 0.97 & 0.435 & 0.05 & $6.1^{(a)}$ & 0.019 & 8 & 1 & \\
\hline HII930 & & 1.18 & 1.39 & 0.14 & 20 & 0.064 & 9 & 1 & \\
\hline HII975 & & 0.77 & 4.082 & 0.05 & 32.0 & 0.224 & 8 & 1 & \\
\hline HII1032 & & 0.70 & 1.299 & 0.12 & $37.2^{(a)}$ & 0.108 & 8 & 3 & $G_{--} f i t$ \\
\hline HII1039 & & 1.18 & $0.85:$ & 0.03 & $5.0^{(a)}$ & 0.039 & 7 & 1 & \\
\hline HII1124 & & 0.94 & 6.051 & 0.07 & $5.6^{(a)}$ & 0.269 & 7 & 1 & \\
\hline HII1136 & V1065 Tau & 0.96 & 0.52 & 0.10 & 60.0 & 0.023 & 2 & 1 & \\
\hline HII1280 & & 1.36 & 0.302 & 0.05 & - & 0.015 & 9 & 1 & \\
\hline HII1305 & & 1.11 & 0.389 & 0.10 & 84 & 0.017 & 9 & 1 & \\
\hline HII1332 & V815 Tau & 0.98 & 8.30 & 0.05 & $2.0^{(a)}$ & 0.363 & 7 & 1 & \\
\hline HII 1512 & & 1.22 & 8.2 & 0.12 & 9 & 0.382 & 9 & 1 & \\
\hline HII1514 & & 0.60 & 3.28 & 0.10 & $13.6^{(a)}$ & 0.360 & 8 & 1 & $G_{--} f i t$ \\
\hline HII1531 & QX Tau & 1.11 & 0.483 & 0.13 & 50.0 & 0.022 & 7 & 1 & \\
\hline HII1532 & & 1.04 & 0.78 & 0.06 & 6: & 0.034 & 9 & 1 & \\
\hline HII1653 & V338 Tau & 1.14 & 0.74 & 0.10 & 21 & 0.034 & 9 & 1 & \\
\hline HII1797 & & 0.52 & 1.73: & 0.01 & $19.6^{(a)}$ & 0.346 & 7 & 1 & \\
\hline HII1883 & V660 Tau & 0.99 & 0.235 & 0.20 & 140.0 & 0.010 & 7 & 3 & \\
\hline HII 2034 & & 0.95 & 0.551 & 0.10 & 75.0 & 0.024 & 7 & 1 & \\
\hline HII 2244 & V664 Tau & 0.95 & 0.565 & 0.17 & 50.0 & 0.025 & 5 & 3 & $K_{-f i t}$ \\
\hline HII 2284 & V1089 Tau & 0.74 & 10.1 & 0.03 & $3.5^{(a)}$ & 0.599 & 6 & 1 & \\
\hline HII 2341 & V1090 Tau & 0.68 & 8.2 & 0.03 & $3.4^{(a)}$ & 0.598 & 6 & 1 & \\
\hline HII 2548 & & 1.29 & 7.5 & 0.06 & 10.0 & 0.358 & 6 & 1 & \\
\hline HII 2741 & & 0.97 & 5.00: & 0.025 & 60.0 & 0.220 & 3 & 1 & \\
\hline HII 2786 & & 0.52 & 2.21 & 0.07 & 25 & 0.441 & 9 & 1 & $F_{--} f i t$ \\
\hline HII2881 & & 0.92 & 4.25 & 0.04 & $7.8^{(a)}$ & 0.191 & 3 & 1 & \\
\hline HII 2927 & V378 Tau & 1.22 & 0.262 & 0.17 & 95.0 & 0.012 & 5 & 2 & \\
\hline HII 2966 & & 1.42 & 4.6 & 0.07 & $\leq 9$ & 0.054 & 9 & 1 & \\
\hline HII3030 & V382 Tau & 1.35 & 7.4: & 0.04 & 10.0 & 0.360 & 6 & 1 & \\
\hline HII3063 & V677 Tau & 1.13 & 0.89 & 0.10 & 26.0 & 0.040 & 6 & 1 & $K_{-f i t}$ \\
\hline HII3163 & V816 Tau & 0.97 & 0.418 & 0.15 & 60.0 & 0.018 & 1 & 2 & $K_{-f i t}$ \\
\hline HII3197 & V679 Tau & 1.03 & 0.44 & 0.04 & 33 & 0.019 & 9 & 1 & \\
\hline
\end{tabular}
(1) Stout-Batalha \& Vogt (1999)
(2) Prosser et al. (1993a)
(3) Prosser et al. (1993b)
(4) Magnitskii (1987)
(5) Stauffer et al. (1987)
(:) uncertain

(6) Prosser et al. (1995)

(7) van Leeuwen et al. (1987)

(8) Marilli et al. (1997)

(9) Krishnamurthi et al. (1998)

(a) Queloz et al. (1998). fitted by a linear law. The $A_{\max }-R_{0}$ relation in the fast rotation range is best fitted by a linear law. The $A_{\max }-R_{0}$ relation in the ultra fast rotation range is best fitted by a power law for G- and K-type stars (Table 2). All the points in the fits were weighted by the number of light curves used to derive them (see Tables 3-8). The correlation coefficients $(r)$, fit parameters $(a$ and $b$ ) and reduced chi-squares $\left(\chi_{\nu}^{2}\right)$ are given in Table 2 .

Since our analysis is mainly focused on main sequence stars, the IC 2602 and IC 2391 members of spectral type 
Table 4. Alpha Persei cluster active stars. The stars that were considered for the $A_{\max }-P$ upper envelope fits are identified in the last column

\begin{tabular}{|c|c|c|c|c|c|c|c|c|c|}
\hline Star & $\begin{array}{l}\text { Other } \\
\text { name }\end{array}$ & $(B-V)_{0}$ & $\begin{array}{l}P_{\text {rot }} \\
\text { (d) }\end{array}$ & $\begin{array}{l}A_{\max } \\
(\mathrm{mag})\end{array}$ & $\begin{array}{l}v \sin i \\
\left(\mathrm{~km} \mathrm{~s}^{-1}\right)\end{array}$ & $R_{0}$ & Ref. & $\begin{array}{c}\# \\
\text { of curves }\end{array}$ & Notes \\
\hline AP15 & & 1.19 & 0.64 & 0.09 & 52.0 & 0.026 & 4 & 1 & \\
\hline AP37 & & 0.86 & 2.40 & 0.03 & 29.0 & 0.114 & 2 & 2 & \\
\hline AP41 & & 0.61 & 5.5 & 0.04 & 9.0 & 0.569 & 9 & 1 & \\
\hline AP43 & & 0.87 & 0.56 & 0.10 & 72.0 & 0.026 & 4 & 1 & \\
\hline AP56 & & 0.90 & 0.36 & 0.14 & 110.0 & 0.016 & 4 & 1 & \\
\hline AP60 & & 1.60 & 0.32 & 0.07 & 105.0 & 0.018 & 3 & 1 & \\
\hline AP63 & & 0.82 & 0.22 & 0.02 & 161.0 & 0.011 & 2 & 1 & \\
\hline AP70 & & 0.90 & 6.4 & 0.09 & 10.0 & 0.292 & 9 & 2 & $K_{--} f i t$ \\
\hline AP72 & & 0.77 & 6.3 & 0.09 & 7.0 & 0.346 & 9 & 1 & $K_{--} f i t$ \\
\hline AP86 & & 1.22 & 0.21 & 0.18 & 140.0 & 0.085 & 7 & 3 & \\
\hline AP91 & & 0.83 & 1.51 & 0.035 & 25.0 & 0.074 & 3 & 1 & \\
\hline AP93 & & 0.83 & 0.62 & 0.095 & 79.0 & 0.030 & 3 & 2 & $K_{--} f i t$ \\
\hline AP95 & & 0.78 & 0.35 & 0.25 & 140.0 & 0.019 & 5 & 2 & $K_{--} f i t$ \\
\hline AP98 & & 0.77 & 6.2 & 0.06 & 7.0 & 0.349 & 9 & 1 & \\
\hline AP100 & & 1.03 & 0.23 & 0.15 & 205.0 & 0.010 & 1 & 3 & \\
\hline AP101 & & 1.14 & 3.2 & 0.2 & 11.0 & 0.133 & 9 & 1 & \\
\hline AP104 & HD 19655 & 0.68 & 0.41 & 0.08 & 10.0 & 0.030 & 8 & 1 & \\
\hline AP114 & & 0.96 & 1.30 & 0.15 & 31.0 & 0.057 & 9 & 1 & \\
\hline AP117 & & 0.85 & 0.47 & 0.17 & 83.0 & 0.022 & 2 & 3 & $K_{--} f i t$ \\
\hline AP118 & HD 19636 & 0.71 & 0.31 & 0.16 & 160.0 & 0.020 & 1 & 3 & \\
\hline $\mathrm{AP} 124$ & & 1.15 & 0.18 & 0.14 & 190.0 & 0.007 & 11 & 2 & \\
\hline $\mathrm{AP} 127$ & HD 232762 & 0.82 & 0.34 & 0.05 & 80.0 & 0.017 & 2 & 1 & \\
\hline AP139 & & 0.80 & 0.26 & 0.12 & 170.0 & 0.013 & 1 & 3 & \\
\hline AP149 & HD 19721 & 0.71 & 0.32 & 0.08 & 117.0 & 0.021 & 2 & 2 & \\
\hline AP156 & & 0.63 & 4.5 & 0.04 & 10.0 & 0.416 & 9 & 1 & \\
\hline AP167 & HD 19805 & 1.13 & 0.41 & 0.10 & 96.0 & 0.017 & 10 & 1 & \\
\hline AP169 & & 0.97 & 5.1 & 0.07 & 8.0 & 0.416 & 9 & 1 & \\
\hline AP193 & & 0.75 & 0.75 & 0.04 & 64.0 & 0.043 & 6 & 1 & \\
\hline AP201 & & 0.94 & 3.75 & 0.07 & 11.0 & 0.167 & 9 & 1 & \\
\hline AP212 & HD 19893 & 0.77 & 7.1 & 0.07 & 6.0 & 0.390 & 9 & 1 & \\
\hline $\mathrm{AP} 225$ & HD 19908 & 0.68 & 0.32 & 0.17 & 138.0 & 0.023 & 8 & 3 & \\
\hline AP 226 & HD 19864 & 0.76 & 0.23 & 0.07 & 181.02 & 0.013 & 2 & 2 & \\
\hline $\mathrm{AP} 244$ & & 0.80 & 0.96 & 0.04 & 42.0 & 0.050 & 2 & 1 & $K_{--} f i t$ \\
\hline AP258 & & 0.73 & 0.26 & 0.10 & 170.0 & 0.016 & 3 & 2 & \\
\hline HE373 & & 0.67 & 0.33 & 0.13 & 140.0 & 0.025 & 2 & 2 & \\
\hline HE520 & V484 Per & 0.69 & 0.29 & 0.09 & 87.0 & 0.020 & 4 & 1 & \\
\hline HE622 & V531 Per & 0.72 & 0.80 & 0.045 & 61.0 & 0.051 & 2 & 2 & \\
\hline HE684 & & 0.47 & 0.75 & 0.065 & 71.0 & 0.243 & 8 & 2 & \\
\hline HE696 & & 0.66 & 0.27 & 0.08 & 10.0 & 0.021 & 8 & 1 & \\
\hline HE699 & V532 Per & 0.61 & 0.33 & 0.10 & 90.0 & 0.034 & 1 & 2 & \\
\hline HE709 & & 0.58 & 0.23 & 0.08 & 59.0 & 0.029 & 8 & 1 & \\
\hline HE935 & & 0.52 & 0.27 & 0.05 & 78.0 & 0.054 & 8 & 1 & \\
\hline
\end{tabular}
(1) O'Dell et al. (1993)
(2) Prosser et al. (1993b)
(3) Prosser et al. (1993a)
(4) Stauffer et al. (1985)
(5) Stauffer et al. (1989)

(6) O’Dell et al. (1997)

(7) Stauffer et al. (1987)

(8) Marilli et al. (1997)

(9) Allain et al. (1996)

(10) Prosser et al. (1995)

(11) Bouvier (1996). later than G8 were excluded from the fitting, because they are pre-main-sequence stars. In the K5-M4 spectral range most of the stars in our sample (all the Alpha Persei and Pleiades as well as a few field stars) have not arrived yet onto the zero-age main-sequence, according to the pre-main-sequence evolutionary tracks computed by D'Antona \& Mazzitelli (1994). That means that our K5M4 sample is not as homogeneous as those discussed before since we have in it stars with quite different convective envelope properties. Therefore, the $A_{\max }$ upper envelope behaviour in this spectral range is not directly comparable with that in the other spectral ranges.

At the first glance, the $A_{\max }$ upper envelope seems to increase at increasing rotation rate up to the highest value of $A_{\max }=0.32 \mathrm{mag}$ at $P \simeq 5$ days $\left(R_{0}=0.06\right)$. Then, it increases less steeply up to a "saturation" level of $A_{\text {max }}=0.20$ mag. However, such break at 5 days, which is only apparent in the K5-M4 spectral range, cannot be considered real since several stars jump from a rotation regime to the other when the Rossby number is used, which is not the case for the 1.1 days boundary.

The smooth onset of the $V$-band variability starting from $R_{0}<1$ and the increase of the upper envelope of the light curves maximum amplitude versus smaller Rossby numbers we found in this study is in apparent contradiction with the results of Hall $(1991,1994)$ and Henry et al. (1995), who found the onset of significant variability at $R_{0}<0.65$ with no obvious increase (decrease) in amplitude down to $R_{0}=0.01$. The apparent contradiction may derive from the use they made of a stellar sample with an extremely large range of global stellar properties. As described in Sect. 3, our analysis instead is focused on a much more homogeneous stellar sample. 
Table 5. Hyades cluster active stars. The stars that were considered for the $A_{\max }-P$ upper envelope fits are identified in the last column

\begin{tabular}{|c|c|c|c|c|c|c|c|c|c|}
\hline Star & $\begin{array}{l}\text { Other } \\
\text { name }\end{array}$ & $(B-V)_{0}$ & $\begin{array}{l}P_{\text {rot }} \\
\text { (d) }\end{array}$ & $\begin{array}{l}A_{\max } \\
(\mathrm{mag})\end{array}$ & $\begin{array}{c}v \sin i \\
\left(\mathrm{~km} \mathrm{~s}^{-1}\right)\end{array}$ & $R_{0}$ & Ref. & $\begin{array}{c}\# \\
\text { of curves }\end{array}$ & Notes \\
\hline VB21 & V984 Tau & 0.816 & 8.98 & 0.038 & - & 0.450 & 2 & 1 & \\
\hline VB25 & V985 Tau & 0.987 & 12.64 & 0.023 & - & 0.554 & 1 & 1 & \\
\hline VB26 & V893 Tau & 0.743 & 9.39: & 0.038 & - & 0.552 & 1 & 1 & \\
\hline VB31 & V986 Tau & 0.566 & 5.44 & 0.049 & 10.0 & 0.752 & 1 & 1 & $F_{--} f i t$ \\
\hline VB43 & V998 Tau & 0.907 & 10.26 & 0.045 & - & 0.466 & 1 & 1 & \\
\hline VB50 & V895 Tau & 0.601 & 7.10 & 0.035 & - & 0.775 & 2 & 1 & $G_{--} f i t$ \\
\hline VB52 & V897 Tau & 0.597 & 7.90 & 0.042 & 6.0 & 0.881 & 2 & 1 & $G_{--} f i t$ \\
\hline VB59 & V992 Tau & 0.543 & 4.98 & 0.032 & 6.0 & 0.820 & 1 & 1 & $F_{--}$fit \\
\hline VB63 & V906 Tau & 0.632 & 7.8 & 0.034 & 7.1 & 0.713 & 2 & 1 & $G_{--} f i t$ \\
\hline VB64 & V911 Tau & 0.657 & 8.67 & 0.035 & 5.1 & 0.699 & 1 & 1 & $G_{--} f i t$ \\
\hline VB69 & V918 Tau & 0.746 & 11.52 & 0.044 & - & 0.672 & 2 & 1 & $K_{--} f i t$ \\
\hline VB73 & V920 Tau & 0.609 & 7.63 & 0.039 & 7.0 & 0.794 & 1 & 1 & $G_{--} f i t$ \\
\hline VB79 & & 0.831 & 11.38 & 0.032 & - & 0.559 & 1 & 1 & \\
\hline VB91 & V996 Tau & 0.883 & 9.36 & 0.051 & - & 0.433 & 1 & 1 & \\
\hline VB92 & V997 Tau & 0.741 & 9.04 & 0.041 & - & 0.534 & 1 & 1 & \\
\hline VB97 & V938 Tau & 0.634 & 8.55 & 0.035 & - & 0.773 & 2 & 1 & $G_{--} f i t$ \\
\hline VB102 & V998 Tau & 0.603 & 6.46 & 0.046 & 7.0 & 0.697 & 1 & 1 & $G_{--} f i t$ \\
\hline VB173 & V989 Tau & 1.237 & 14.14 & 0.034 & - & 0.569 & 1 & 1 & \\
\hline VB175 & V991 Tau & 1.031 & 10.82 & 0.036 & - & 0.465 & 1 & 1 & \\
\hline VB174 & V990 Tau & 1.058 & 11.88 & 0.032 & - & 0.507 & 1 & 1 & \\
\hline VB181 & V995 Tau & 1.167 & 11.92 & 0.040 & - & 0.491 & 1 & 1 & \\
\hline
\end{tabular}

(1) Radick et al. (1987)

(:) uncertain period.

(2) Lockwood et al. (1984)

Table 6. IC 2391 cluster active stars (Patten \& Simon 1996). The stars that were considered for the $A_{\max } P$ upper envelope fits are identified in the last column

\begin{tabular}{|c|c|c|c|c|c|c|c|c|}
\hline VXR & $\begin{array}{l}\text { Other } \\
\text { name }\end{array}$ & $(B-V)_{0}$ & $\begin{array}{l}P_{\text {rot }} \\
\text { (d) }\end{array}$ & $\begin{array}{l}A_{\max } \\
(\mathrm{mag})\end{array}$ & $\begin{array}{c}v \sin i \\
\left(\mathrm{~km} \mathrm{~s}^{-1}\right)\end{array}$ & $R_{0}$ & $\begin{array}{c}\# \\
\text { of curves }\end{array}$ & Notes \\
\hline 12 & SHJM 6 & 0.83 & 3.86 & 0.07 & 16 & 0.189 & 2 & \\
\hline 14 & & 0.56 & 1.32 & 0.08 & - & 0.191 & 2 & $F_{-} f i t$ \\
\hline $35 a$ & & 0.99 & 0.527 & 0.15 & 90 & 0.023 & 1 & \\
\hline $38 \mathrm{a}$ & SHJM 3 & 1.23 & 2.78 & 0.10 & 18 & 0.111 & 2 & \\
\hline 41 & SHJM 8 & 1.25 & 5.80 & 0.22 & $\leq 15$ & 0.231 & 2 & \\
\hline $42 \mathrm{a}$ & SHJM 9 & 1.54 & 1.81 & 0.05 & - & 0.066 & 1 & \\
\hline $45 \mathrm{a}$ & & 0.80 & 0.223 & 0.07 & - & 0.011 & 1 & \\
\hline 47 & & 1.43 & 0.258 & 0.13 & 95 & 0.002 & 1 & \\
\hline $60 \mathrm{a}$ & SHJM 10 & - & 0.93: & 0.09 & $<15$ & - & 1 & \\
\hline $60 \mathrm{~b}$ & SHJM 4 & - & 0.212 & 0.10 & $\overline{1} 50$ & - & 1 & \\
\hline $62 \mathrm{a}$ & SHJM 5 & 0.85 & $0.503:$ & 0.12 & - & 0.024 & 1 & \\
\hline $64 a$ & & - & 0.543 & 0.05 & - & - & 1 & \\
\hline 70 & & 0.63 & 2.67 & 0.08 & - & 0.246 & 1 & $G_{--} f i t$ \\
\hline 72 & & 0.72 & 3.23 & 0.08 & - & 0.203 & 1 & \\
\hline $76 \mathrm{a}$ & & 1.04 & 4.86 & 0.14 & - & 0.208 & 1 & \\
\hline $77 \mathrm{a}$ & & 0.50 & 0.653 & 0.07 & - & 0.177 & 1 & \\
\hline
\end{tabular}

(:) uncertain period.

The existence of a discontinuity in passing from ultra fast to fast rotating stars may be attributed to an abrupt change of the total spotted areas or of their spatial redistribution and would imply different efficiency or different operation mode of the dynamo action when operating at rather different rotation regimes.

\subsection{Saturation}

Chromospheric, TR and coronal emission fluxes show evidence of saturation, i.e., a progressive flattening of their activity rate increase with advancing rotation rate, at $R_{0} \simeq 0.33$ for chromospheric fluxes and at slightly larger values, $R_{0} \simeq 0.5-0.7$, for $\mathrm{TR}$ and coronal fluxes (Vilhu 1984).
Several causes may be responsible for the observed saturation, such as:

a) the filling of the entire atmosphere by magnetic regions $(f \rightarrow 1)$;

b) a negative feedback on the convective motions and on the differential rotation due to the widespread presence of intense magnetic fields (Robinson \& Durney 1982);

c) a saturation of the emission mechanism efficiency.

We find evidence that the upper envelope of the $A_{\text {max }}-P$ correlation stops increasing at rotation periods $P \lesssim 0.35$ days (Fig. 5) and, similar to the results of 
Table 7. IC 2602 cluster active stars (Barnes et al. 1999). The stars that were considered for the $A_{\max }-P$ upper envelope fits are identified in the last column

\begin{tabular}{lllccccc}
\hline \hline Name & $(B-V)_{0}$ & $\begin{array}{l}P_{\text {rot }} \\
(\mathrm{d})\end{array}$ & $\begin{array}{c}A_{\max } \\
(\mathrm{mag})\end{array}$ & $\begin{array}{c}v \sin i \\
\left(\mathrm{~km} \mathrm{~s}^{-1}\right)\end{array}$ & $R_{0}$ & $\begin{array}{c}\# \\
\text { of curves }\end{array}$ & Notes \\
\hline B134 & 0.91 & 6.8 & 0.025 & 10 & 0.308 & 1 \\
W79 & 0.79 & 6.2 & 0.02 & 8 & 0.326 & 1 \\
R15 & 0.89 & $3.6:$ & 0.01 & 7 & 0.166 & 1 \\
R24A & 1.39 & 1.25 & 0.06 & 34 & 0.018 & 1 \\
R26 & 1.50 & 5.7 & 0.05 & 6 & 0.044 & 1 \\
R27 & 1.46 & $4.5:$ & 0.035 & 10 & 0.166 & 1 \\
R29 & 1.07 & 2.21 & 0.05 & 22 & 0.042 & 1 \\
R31 & 1.55 & 0.49 & 0.02 & 35 & 0.003 & 1 \\
R32 & 1.59 & 4.0 & 0.075 & 9 & 0.023 & 1 \\
R43 & 0.90 & 0.78 & 0.035 & 50 & 0.035 & 1 \\
R44 & 1.50 & 5.5 & 0.04 & 7 & 0.041 & 1 \\
R50 & 1.51 & 6.4 & 0.02 & 7 & 0.046 & 1 \\
R52 & 1.02 & 0.393 & 0.07 & 95 & 0.017 & 1 \\
R53B & 1.57 & 0.41 & 0.045 & 100 & 0.002 & 1 \\
R56 & 1.39 & 4.1 & 0.055 & 17 & 0.060 & 1 \\
R57 & 1.56 & 8.7 & 0.02 & 6 & 0.054 & 1 \\
R58 & 0.60 & 0.57 & 0.055 & 93 & 0.059 & 1 \\
R66 & 0.64 & 3.3 & 0.035 & 12 & 0.289 & 1 \\
R70 & 0.65 & 4.3 & 0.02 & 11 & 0.359 & 1 \\
R72 & 0.59 & 1.05 & 0.035 & 49 & 0.115 & 1 \\
R77 & 1.43 & 10.1 & 0.03 & 7 & 0.111 & 1 \\
R83 & 0.58 & 1.67 & 0.035 & 30 & 0.209 & 1 \\
R88A & 1.16 & 0.204 & 0.04 & 200 & 0.008 & 1 \\
R89 & 1.20 & 4.8 & 0.065 & 14 & 0.195 & 1 \\
R92 & 0.63 & $2.0:$ & 0.015 & 14 & 0.185 & 1 \\
R93 & 1.33 & 6.7 & 0.025 & 8 & 0.262 & 1 \\
R94 & 1.35 & 2.6 & 0.035 & 23 & 0.101 & 1 \\
R95A & 0.83 & 1.20 & 0.04 & 12 & 0.059 & 1 \\
R96 & 1.21 & 1.82 & 0.05 & 17 & 0.074 & 1 & \\
\hline \hline & & & & & & & \\
\end{tabular}

(:) uncertain period.

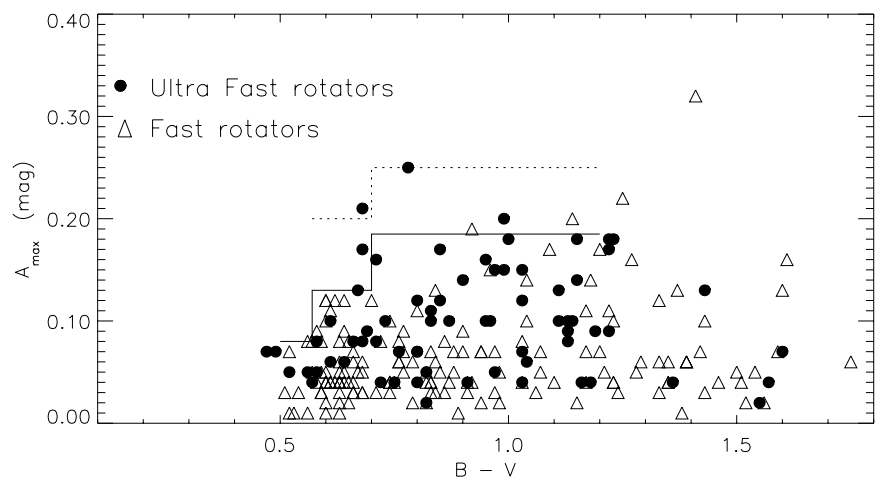

Fig. 6. The light curve maximum amplitude versus $B-V$ colour. Continuous and dotted horizontal lines indicate the offset values (parameter $a$ in Table 2) of the linear fits to the $A_{\max }-P$ relation for fast and ultra fast stars, respectively

O'Dell et al. (1995), at Rossby numbers $R_{0} \lesssim 0.018$. The lack of correlation between $A_{\max }$ and $P$, at very high rotation rates may be simply due to an underestimated $A_{\max }$ upper envelope, since it is a lower limit. However, as is clear when $R_{0}$ is used (Fig. 4; see also O'Dell et al. 1995), we may tentatively infer the presence of "saturation". By this, we mean that either the dynamo stops producing more spots, or it produces additional spots in such a way that $A_{\text {max }}$ does not increase (e.g., by evenly distributing them).

If our hypothesis of a saturation effect is correct, since such saturation appears at values of $R_{0}\left(R_{0}<0.02\right)$ orders of magnitude smaller than the values at which chromospheric, TR's and coronal emission fluxes saturate $\left(R_{0}<0.33\right)$, the spot covering fraction appears to be a more suitable indicator of the magnetic dynamo activity at $R_{0}$ values $\left(0.02<R_{0}<0.33\right)$, i.e. below the values where activity flux saturation occurs. The lack of detection of a saturation regime by Krisnamurthi et al. (1998) may possibly be due to the less numerous fast rotators they had in their stellar sample.

\subsection{Color and age dependence}

As shown in Figs. 3 and 6, the photospheric activity level measured by the maximum light curve amplitude depends on the star's color. Both ultra fast and fast rotating K-type stars reach an activity level (as shown by the data upper envelope) higher than F-, and G-type stars of same rotation period. The dependence on the $B-V$ colour (Fig. 6) is very similar in both rotation ranges. Continuous and dotted horizontal lines indicate the offset values (parameter $a$ in Table 2) of the linear fits to the $A_{\max }-P$ relation for fast and ultra fast stars, respectively.

The cluster ages we are considering in our sample are in the range $0.03 \mathrm{Gyr}$ (IC 2602 and IC 2391 clusters) to 0.6 Gyr (Hyades cluster). As shown in Fig. 3, in the F, G and $\mathrm{K}$ spectral range the value of the upper envelope is determined mainly by the Pleiades cluster and field stars, most of which belong to the Pleiades cluster moving group. Therefore, it seems that the spot activity level on the main sequence increases from the zero-age main sequence, with its maximum level around the Pleiades age (0.07 Gyr), and 
Table 8. Field active stars. The stars that were considered for the $A_{\max }-P$ upper envelope fits are identified in the last column

\begin{tabular}{|c|c|c|c|c|c|c|c|c|c|}
\hline Star & $\begin{array}{l}\text { Other } \\
\text { name }\end{array}$ & $B-V$ & $\begin{array}{r}P_{\text {rot }} \\
\text { (d) }\end{array}$ & $\begin{array}{l}A_{\max } \\
(\mathrm{mag})\end{array}$ & $\begin{array}{c}v \sin i \\
\left(\mathrm{~km} \mathrm{~s}^{-1}\right)\end{array}$ & $R_{0}$ & Ref. & $\begin{array}{c}\# \\
\text { of curves }\end{array}$ & Notes \\
\hline HD 987 & & 0.71 & 4.2 & 0.03 & 10 & 0.273 & 17 & 1 & \\
\hline HD $1835^{a}$ & BE Cet & 0.66 & 7.655 & 0.05 & - & 0.609 & 20,2 & 25 & $G_{-f i t}$ \\
\hline HD 7172 & & 0.63 & 3.1 & 0.06 & 13 & 0.286 & 17 & 1 & \\
\hline HD 17925 & EP Eri & 0.87 & 6.57 & 0.04 & $\leq 8$ & 0.308 & 2 & 3 & \\
\hline HD 18134 & VZ Hor & 0.74 & 2.0 & 0.10 & - & 0.135 & 11 & 1 & \\
\hline HD 18632 & BZ Cet & 0.66 & 10.22 & 0.02 & 1.8 & 0.813 & 13 & 1 & $G_{-f i t}$ \\
\hline HD 20630 & $\kappa^{1}$ Cet & 0.68 & 9.0 & 0.03 & 3.8 & 0.656 & 2 & 3 & \\
\hline HD 21845 & V577 Per & 0.79 & 1.454 & 0.06 & - & 0.076 & 2 & 3 & \\
\hline HD 25893 & V491 Per & 0.85 & 7.37 & 0.05 & 4.5 & 0.353 & 12 & 3 & \\
\hline HD 26913 & V891 Tau & 0.68 & 6.8 & 0.06 & 3.9 & 0.496 & 8 & 4 & $G \_f i t$ \\
\hline HD 29697 & V834 Tau & 1.09 & 3.936 & 0.17 & 9.5 & 0.167 & 13 & 2 & $K_{-f i t}$ \\
\hline HD 31993 & V1192 Ori & 1.28 & 6.78 & 0.05 & 31.1 & 0.269 & 14 & 1 & \\
\hline HD 35296 & V111 Tau & 0.53 & 3.6 & 0.01 & 15.4 & 0.660 & 29,10 & 2 & \\
\hline HD 36705 & AB Dor & 0.83 & 0.5146 & 0.11 & 85 & 0.025 & 14,1 & 6 & $K_{-f i t}$ \\
\hline HD 36869 & & 0.64 & 1.31 & 0.09 & 28 & 0.140 & 22,1 & 2 & $G_{-f i t}$ \\
\hline HD 37394 & & 0.84 & 10.00 & 0.03 & 4.0 & 0.485 & 2 & 1 & \\
\hline HD 37572 & UY Pic & 0.82 & 4.3: & 0.05 & 9 & 0.214 & 1 & 1 & \\
\hline HD $39587^{a}$ & $\chi^{1}$ Ori & 0.59 & 5.24 & 0.03 & 9 & 0.621 & 2 & 21 & $G_{-f i t}$ \\
\hline HD 42807 & & 0.66 & 7.8 & 0.03 & - & 0.621 & 10 & 1 & \\
\hline HD 43989 & V1358 Ori & 0.57 & 1.15 & 0.08 & 47 & 0.154 & 22 & 2 & $F_{--} f i t$ \\
\hline HD 45081 & AO Men & 1.20 & 2.65 & 0.17 & 17 & 0.108 & 1 & 2 & \\
\hline HD 52452 & & 0.68 & 0.423 & 0.21 & 15 & 0.031 & 2 & 6 & $G_{-f i t}$ \\
\hline HD 64096 & 9 Pup & 0.60 & 9.7 & 0.02 & 8 & 1.066 & 10 & 1 & $G_{-f i t}$ \\
\hline HD 70573 & & 0.63 & 3.296 & 0.05 & 13.6 & 0.304 & 13 & 1 & \\
\hline HD $72905^{a}$ & $\pi^{1} \mathrm{Uma}$ & 0.60 & 4.5395 & 0.04 & 9.5 & 0.502 & 2 & 10 & $G_{-f i t}$ \\
\hline HD 78644 & & 0.64 & 4.6: & 0.05 & 59 & 0.402 & 1 & 1 & \\
\hline $\mathrm{HD} 82443^{a}$ & DX Leo & 0.76 & 5.377 & 0.08 & 6.5 & 0.304 & 9 & 15 & $K_{-f i t}$ \\
\hline $\mathrm{HD} 82558^{a}$ & LQ Hya & 0.92 & 1.61 & 0.19 & 26 & 0.073 & 15 & 23 & $K_{-} f i t$ \\
\hline HD 88230 & & 1.38 & 6.0: & 0.01 & 3.1 & 0.095 & 3 & 1 & \\
\hline HD 95650 & DS Leo & 1.43 & 2.94 & 0.10 & - & 0.033 & 16 & 1 & \\
\hline HD 98712 & SZ Crt & 1.36 & 11.58 & 0.06 & - & 0.448 & 23,1 & 5 & \\
\hline HD 114710 & $\beta$ Com & 0.56 & 12.26 & 0.01 & 4.3 & 1.771 & 4 & 1 & $F_{--f i t}$ \\
\hline HD $115383^{a}$ & $59 \mathrm{Vir}$ & 0.59 & 3.328 & 0.03 & 3 & 0.386 & 2 & 19 & $G_{-f i t}$ \\
\hline HD 115404 & & 0.94 & 18.8 & 0.02 & 3.9 & 0.838 & 3 & 1 & \\
\hline HD 118100 & EQ Vir & 1.17 & 4.03 & 0.11 & 13 & 0.164 & 21,19 & 4 & \\
\hline HD $129333^{a}$ & EK Dra & 0.61 & 2.787 & 0.10 & - & 0.283 & 2 & 26 & $G_{-f i t}$ \\
\hline $\mathrm{HD} 134319^{a}$ & IU Dra & 0.68 & 4.448 & 0.04 & - & 0.324 & 6 & 10 & $G_{-f i t}$ \\
\hline HD 140637 & KW Lup & 1.02 & 2.72 & 0.11 & 14 & 0.119 & 22 & 1 & \\
\hline HD 149661 & $12 \mathrm{Oph}$ & 0.82 & 11.0 & 0.02 & 0.6 & 0.549 & 18 & 1 & \\
\hline HD $152391^{a}$ & V2292 Oph & 0.76 & 11.45 & 0.06 & 3.7 & 0.644 & 2 & 21 & $K_{-f i t}$ \\
\hline HD 156026 & V2215 Oph & 1.15 & 21.0: & 0.02 & 2.2 & 0.869 & 14 & 1 & \\
\hline HD 160934 & & 1.23 & 1.842 & 0.02 & 16.4 & 0.074 & 13 & 1 & \\
\hline HD 171488 & V889 Her & 0.60 & 1.341 & 0.12 & 40 & 0.123 & 2 & 6 & $G_{-f i t}$ \\
\hline HD 190406 & 15 Sge & 0.60 & 13.95 & 0.01 & 4 & 1.443 & 2 & 3 & $G_{-f i t}$ \\
\hline HD 197481 & AU Mic & 1.44 & 4.9 & 0.32 & - & 0.061 & 24,7 & 10 & \\
\hline HD 206860 & HN Peg & 0.59 & 4.70 & 0.05 & 11 & 0.550 & 2 & 2 & \\
\hline HD 216803 & TW Psa & 1.10 & 10.3 & 0.04 & - & 0.433 & 5 & 1 & \\
\hline HD $234601^{a}$ & & 0.63 & 7.35 & 0.04 & 7 & 0.679 & 30 & 9 & $G_{-f i t}$ \\
\hline HD 295290 & & 0.58 & 3.04: & 0.09 & - & 0.380 & 1 & 1 & $G_{-f i t}$ \\
\hline $\mathrm{SAO} 45568$ & & 0.59 & 8.13 & 0.06 & - & 0.942 & 2 & 10 & $G_{-f i t}$ \\
\hline SAO 51891 & V383 Lac & 0.86 & 2.42 & 0.08 & 19.8 & 0.114 & 13 & 1 & \\
\hline \multirow[t]{5}{*}{ SAO 150508} & & 0.67 & 1.895 & 0.05 & 20 & 0.144 & 1 & 1 & \\
\hline & GT Peg & 1.6 & 1.64 & 0.13 & - & 0.009 & 25,7 & 1 & \\
\hline & V1005 Ori & 1.37 & 1.90 & 0.13 & - & 0.038 & 26,7 & 1 & \\
\hline & V1285 Aql & 1.75 & 2.9 & 0.06 & - & 0.011 & 27,7 & 1 & \\
\hline & YZ CMi & 1.61 & 2.8 & 0.16 & - & 0.015 & 28,7 & 1 & \\
\hline EXO 052707 & -3329.2 & 1.07 & 2.22 & 0.07 & 40 & 0.094 & 17 & 2 & \\
\hline 1E2349.8-0112 & BS Psc & 0.62 & 1.145 & 0.12 & 47.3 & 0.111 & 13 & 1 & \\
\hline HE 628 & & 0.65 & 2.676: & 0.045 & - & 0.230 & 31 & 1 & \\
\hline HE 1150 & & 0.62 & 5.64 & 0.08 & - & 0.470 & 31 & 1 & $G_{-f i t}$ \\
\hline
\end{tabular}

(1) Cutispoto \& Messina, person. comm.

(2) Messina 1998, Ph.D. Thesis

(3) Chugainov (1991)

(4) Dorren et al. (1993), person. comm

(5) Vogt (1975)

(6) Messina et al. (1999a)

(7) Alekseev \& Gershberg (1996)

(8) Ziegler et al. (1984)

(9) Messina et al. (1999b)

(10) Stepien \& Geyer (1996)

(a) star in Fig. 2
(11) Eggen (1984)

(12) Strassmeier et al. (1989)

(13) Henry et al. (1995)

(14) Lloyd Evans \& Koen (1987)

(15) Oláh et al. (2000)

(16) Bopp et al. (1983)

(17) Cutispoto et al. (1996)

(18) Dorren \& Guinan (1982)

(19) Anderson (1979)

(20) Chugainov (1980)

(:) uncertain period
(21) Torres \& Ferraz-Mello (1971)

(22) Cutispoto et al. (1999)

(23) Torres et al. (1985)

(24) Torres et al. (1972)

(25) Bopp \& Evans (1973)

(26) Bopp et al. (1978)

(27) Byrne et al. (1984)

(28) Chugainov (1974)

(29) Donahue (1993)

(30) Cutispoto et al. (2000) in press

(31) Marilli et al. (1997). 
then decreases. In the $\mathrm{M}$ spectral range, where most of the stars are in the pre-main sequence evolutionary stage, the spot activity level appears to be somehow higher than on the main sequence.

\subsection{Activity-flux relation}

Activity diagnostics formed at various temperature regimes or atmospheric levels are strongly correlated each other. The flux-flux relations are best fitted by power laws with steepening slopes when increasingly high temperature diagnostics are plotted against the coolest ones (Rutten et al. 1991; Piters et al. 1997; Ayres et al. 1995; Marilli \& Catalano 1984). These relations still hold when stars of different spectral type, luminosity class and multiplicity are considered, indicating that the activity structures are temporally and possibly spatially correlated at all atmospheric levels. These correlations are similar for a wide variety of stellar parameters.

The dependence of spot activity upper envelope on the rotation period for "fast" G stars was then compared with the rotational dependence of other activity indicators in the corona, TR and chromosphere: X-ray, Mg II h\&k and C IV luminosity, respectively. In our analysis we used the luminosity-rotation relations of Dorren et al. (1994), because all the stars we used to define the upper envelope of G-type fast stars (last column of Tables 3-8) have the same global properties (G0-G7 spectral range, rotation period longer than $\sim 1.1$ days, and age of the Pleiades, or older) of those analysed by Dorren et al. within the "Sun in Time" project at the Villanova University (Dorren et al. 1994; Dorren \& Guinan 1994; Guinan et al. 1995) and from which the relations between X-ray, Mg II h\&k and C IV luminosity and rotation period were derived. Hence, most of the best observed G-type fast stars were selected just from the "Sun in Time" stellar sample. The following mean relations are found to apply:

$$
\begin{aligned}
& L_{\mathrm{X}} \quad \propto P^{-2.01} \\
& L_{\mathrm{CIV}} \propto P^{-1.50} \\
& L_{\mathrm{MgII}} \propto P^{-0.94} \\
& A_{\max } \propto P^{-0.58} \quad(\text { G-type fast stars }) .
\end{aligned}
$$

The relations (3), (4) and (5) were derived from the data in Table 3 of Dorren et al. (1994), the relation (6) is from Table 2 .

On the basis of the strong correlation between the photospheric and upper layers' activity diagnostics and the rotation period, we expect that a relation should also exist between the $A_{\max }$ upper envelope and the upper layers' activity diagnostics. By folding Eqs. (3-6) we may predict the following relations:

$$
\begin{aligned}
& L_{\mathrm{X}} \propto A_{\max }^{3.41} \\
& L_{\mathrm{CIV}} \propto A_{\max }^{2.54}
\end{aligned}
$$

$$
L_{\mathrm{MgII}} \propto A_{\max }^{1.59}
$$

or

$L_{\mathrm{X}} \propto L_{\mathrm{CIV}}^{1.34} \propto L_{\mathrm{MgII}}^{2.14} \propto A_{\max }^{3.41}$.

However, it must be stressed that such relations are just predicted and must be considered very tentative since they are folded and obtained by substituting the upper envelope relation for our sample of fast G0-G7 type stars into relations for a much larger sample of G0-G8 stars, of which our upper envelope fast stars are only a small fraction.

\section{Conclusion}

We have presented an extension down to the photosphere of the rotation-activity connection, so far extensively studied and well established for the outermost atmospheric layers. It is shown that the envelope of the maximum light curve amplitude in the $V$-band $\left(A_{\max }\right)$ can be adopted as an indicator of a lower limit of the photospheric magnetic filling factor $\left(f_{\mathrm{S}}\right)$ and, therefore, as a measure of a lower limit of the photospheric magnetic activity confined into spots. The study of the photospheric magnetic activity by means of $A_{\max }$ in a sample of 225 field and cluster active stars, which is much larger than previously analysed (O'Dell et al. 1995; Krishnamurthi et al. 1998), reveals the existence of different behaviours of the $A_{\max }-P$ correlation in different rotation rate ranges. At the base of these behaviours may reside a different dynamo efficiency, which effectively operates within a limited rotation range. Moreover, the absence of correlation in the $A_{\max }-P$ relation, at very high rotation rates, and Rossby numbers $R_{0} \leq 0.02$, may be tentatively interpreted as a saturation effect. We believe that saturation is a regime in which the dynamo can produce the widest range of distribution of $A_{\max }$ values from very small to maximum. In the case the hypothesis of the existence of a saturation regime is correct, since the value of $R_{0}$ at which $A_{\max }$ saturates is orders of magnitude smaller than the value at which chromospheric, TR's and coronal emission fluxes saturate, the spot covering fraction would be the most reliable indicator of magnetic dynamo activity at rotation rates above those where activity fluxes saturate.

The activity level, as measured by the envelope of the maximum light curve amplitudes, appears to depend also on the $B-V$ colour and age. The maximum activity level is found in the $\mathrm{K}$ spectral range and at the Pleiades cluster age, for both fast and ultra fast stars. The remarkable photospheric activity level on $\mathrm{K}$ stars, relative to other spectral types, has been suggested more than two decades, when early evidence concerning a small sample of active RS CVn-type binaries was presented (Catalano et al. 1980). Our results are based on a much larger stellar sample. Hence, we can conclude with confidence that the highest dynamo efficiency, at least in producing asymmetric spot distributions, and the best observation conditions for photospheric activity development and manifestations at the main sequence age are met in K-type stars. 
At the pre-main sequence evolutionary stage the spot activity level appears somehow higher than for main sequence stars.

On the basis of the strong correlation between the photospheric and upper layers' activity diagnostics and the rotation period, we tentatively predict that a relation should also exist between the $A_{\max }$ upper envelope and the upper layers' activity diagnostics (see Eq. (10)). This aspect certainly deserves to be more deeply investigated and tested by using data in the literature and new data bases.

Acknowledgements. The authors are very grateful to the referee Dr. S. Saar for very careful reading and several helpful suggestions for improving the manuscript. S. Messina is also grateful to Dr. L. Girardi for kindly computing and making available to us the internal structure models for M-type stars.

The extensive use of the SIMBAD and ADS databases operated by the CDS center, Strasbourg, France, is also gratefully acknowledged.

Active star research at the Department of Physics and Astronomy of Catania University and Catania Astrophysical Observatory is funded by MURST (Ministero dell'Università e della Ricerca Scientifica e Tecnologica), CNAA (Consorzio Nazionale per l'Astronomia e l'Astrofisica) and the Regione Siciliana, whose financial support is gratefully acknowledged.

\section{References}

Alekseev, I. Y., \& Gershberg, R. E. 1996, Astron. Reports, 40, 538

Allain, S., Fernandez, M., Martin, E. L., \& Bouvier, J. 1996, A\&A, 314, 173

Anderson, C. M. 1979, PASP, 91, 202

Ayres, T. R., Fleming, T. A., Simon, T., et al. 1995, ApJS, 96 223

Barnes, S. A., Sofia, S., Prosser, C. F., \& Stauffer, J. R. 1999, ApJ, 516, 263

Bopp, B. W., \& Evans, D. S. 1973, MNRAS, 154, 343

Bopp, B. W., Torres, C. A. O., Busko, I. C., \& Quast, G. R. 1978, IBVS, 1443

Bopp, B. W., Africano, J. L., Stence, R. E., Noha, P. V., \& Klimke, A. 1983, AJ, 275, 691

Bouvier, J. 1996, A\&AS, 120, 127

Byrne, P. B., Doyle, J. G., Butler, C. J., \& Andrews, A. D. 1984, MNRAS, 211, 607

Byrne, P. B., Panagi, P. M., Lanzafame, A. C., et al. 1995, A\&A, 299, 115

Catalano, S., Frisina, A., \& Rodonò, M. 1980, in Symp. IAU 88, Close binary stars: Observations and interpretation, ed. M. J. Plavec, D. M. Popper, \& R. K. Ulrich (Dordrecht: Reidel), 405

Chugainov, P. F. 1974, Izv. Krimskoi Astrofiz. Obs., 54, 3

Chugainov, P. F. 1980, Izv. Krimskoi Astrofiz. Obs., 61, 124

Chugainov, P. F. 1991, in Angular Momentum Evolution of Young Stars, ed. S. Catalano, \& R. J. Stauffer (Kluwer), 175

Cutispoto, G., Tagliaferri, G., Pallavicini, R., Pasquini, L., \& Rodonò, M. 1996, A\&AS, 115, 41

Cutispoto, G., Pastori, L., Tagliaferri, G., Messina, S., \& Pallavicini, R. 1999, A\&AS, 138, 87
Cutispoto, G., Pastori, L., Guerrero, A., et al. 2000, A\&A, in press

D'Antona, F., \& Mazzitelli, I. 1994, ApJS, 90, 467

Donahue, R. 1993, Ph.D. Thesis, New Mexico State University

Dorren, J. D., \& Guinan, E. F. 1982, AJ, 87, 1546

Dorren, J. D., \& Guinan, E. F. 1994, ApJ, 428, 805

Dorren, J. D., Guinan, E. F., \& DeWarf, L. E. 1994, in ASP Conf. Ser. 64, Cool stars, Stellar Systems and the Sun, ed. J.-P. Caillaut, 399

Eggen, O. J. 1984, AJ, 89, 1358

Girardi, L., Bressan, A., Bertelli, G., \& Chiosi, C. 2000, A\&AS, 141,371

Guinan, E. F., DeWarf, L. E., Messina, S., \& McCook, G. P. 1995, BAAS, 186, 2101

Hall, D. S. 1991, in Coll. IAU 130, The Sun and Cool Stars. Activity, Magnetism and Dynamos, ed. I. Tuominen, D. Moss, \& G. Rudiger (Springer-Verlag), 353

Hall, D. S. 1994, Mem. Soc. Astron. It., 65, 73

Henry, G. W., Fekel, F. C., \& Hall, D. S. 1995, AJ, 110, 2926

Kopp, G., \& Rabin, D. 1992, Sol. Phys., 141, 253

Krishnamurthi, A., Terndrup, D. M., Pinsonneault, M. H., et al. 1998, ApJ, 493, 914

Lanza, A. F., Catalano, S., Cutispoto, G., Pagano, I., \& Rodonò, M. 1998, A\&A, 332, 541

Lloyd Evans, T., \& Koen, M. C. J. 1987, South Afr. Obs. Circ., 11,21

Lockwood, G. W., Thompson, D. T., Radick, R. R., et al. 1984, PASP, 96, 714

Magnitskii, A. K. 1987, Sov. Astron. Lett., 13, 451

Marilli, E., \& Catalano, S. 1984, A\&A, 133, 57

Marilli, E., Catalano, S., \& Frasca, A. 1997, Mem. Soc. Astron. Ital., 68, 895

Mathys, G. 1989, Fundamen. Cosm. Phys., 13, 143

Messina, S. 1998, Ph.D. Thesis, Univ. of Catania, Italy

Messina, S., Guinan, E. F., \& Lanza, A. F. 1999a, Ap\&SS, 260, 493

Messina, S., Guinan, E. F., Lanza, A. F., \& Ambruster, C. 1999b, A\&A, 347, 249

Messina, S. 2000, submitted to A\&A

Neff, J. E., O'Neal, D., \& Saar, S. H. 1995, ApJ, 452, 879

Noyes, R. W., Hartmann, L., Baliunas, S. L., Dunkan, D. K., \& Vaughan, A. H. 1984, ApJ, 279, 763

O'Dell, M. A., \& Collier Cameron, A. 1993, MNRAS, 262, 521

O'Dell, M. A., Panagi, P., Hendry, M. A., \& Collier Cameron, A. 1995, A\&A, 294, 715

O'Dell, M. A., Hilditch, R. W., Collier Cameron, A., \& Bell, S. A. 1997, MNRAS, 284, 874

Oláh, K., Kolláth, Z., \& Strassmeier, K. G. 2000, A\&A, 356, 643

O'Neal, D., Saar, S. H., \& Neff, J. E. 1996, ApJ, 463, 766

O'Neal, D., Saar, S. H., \& Neff, J. E. 1998, ApJ, 501, L73

Pallavicini, R. 1992, in The Sun as a Laboratory for Astrophysics, ed. J. T. Schmeltz, \& J. C. Brown (Dordrecht: Kluwer), 313

Parker, E. N. 1979, Cosmic Magnetic Fields, their Origin and Activity (Oxford: Clarendon Press)

Patten, B. M., \& Simon, T. 1996, ApJS, 106, 489

Pinsonneault, M. H., Stauffer, J., Soderblom, D. R., King, J. R., \& Hanson, R. B. 1998, ApJ, 504, 170

Piters, A. J. M., Schrijver, C. J., Schmitt, J. H. M. M., et al. 1997, A\&A, 325, 1115

Prosser, C. F., Schild, R. E., Stauffer, J. R., \& Jones, F. J. 1993a, PASP, 105, 269 
Prosser, C. F., Shetrone, M. D., Marilli, E., et al. 1993b, PASP, 105, 1407

Prosser, C. F., Dasgupta, A., Baknam, D. E., et al. 1995, PASP, 107,211

Queloz, D., Allain, S., Mermilliod, J.-C., Bouvier, J., \& Mayor, M. 1998, A\&A, 335, 183

Radick, R. R., Thompson, D. T., Lockwood, G. W., Dunkan, D. K., \& Baggett, W. E. 1987, ApJ, 321, 459

Radick, R. R., Lockwood, G. W., \& Baliunas, S. L. 1989, Science, 247, 39

Radick, R. R., Lockwood, G. W., Skiff, B. A., \& Baliunas, S. L. 1998, ApJS, 118, 239

Robinson, R. D., \& Durney, B. R. 1982, A\&A, 108, 322

Rodonò, M., Lanza, A. F., \& Catalano, S. 1995, A\&A, 301, 75

Rutten, R. G. M., Schrijver, C. J., Lemmens, A. F. P., \& Zwaan, C. 1991, A\&A, 252, 203

Saar, S. H. 1990, in Solar Photosphere: Structure, Convection and Magnetic Fields, ed. J. O. Stenflo (Dordrecht: Kluwer), 427

Saar, S. H. 1996, in Coll. IAU 153, Magnetodynamics Phenomena in the Solar atmosphere - Prototypes of Stellar Magnetic Activity, ed. Y. Uchida, T. Kosugi, \& H. S. Hudson (Kluwer), 367

Schüssler, M. 1983, in Symp. IAU 102, Solar and stellar magnetic fields, ed. J. O. Stenflo (Dordrecht: Reidel), 213

Stauffer, J. R., Hartmann, L. W., Birnham, J. N., \& Jones, B. F. 1985, ApJ, 289, 247

Stauffer, J. R., Schild, R. E., Baliunas, S. L., \& Africano, J. L. 1987, PASP, 99, 471

Stauffer, J. R., Hartmann, L. W., \& Jones, B. F. 1989, ApJ, 346,160

Stepien, K., \& Geyer, E. 1996, A\&AS, 117, 83

Stout-Batalha, N. M., \& Vogt, S. S. 1999, ApJS, 123, 251

Strassmeier, K. G., Hall, D. S., Boyd, L. J., \& Genet, R. M. 1989, ApJS, 69, 141

Torres, C. A. O., \& Ferraz-Mello, S. 1971, IBVS, 577

Torres, C. A. O., Ferraz-Mello, S., \& Quast, G. R. 1972, Astrophys. Lett., 11, 13

Torres, C. A. O, Busko, I. C., \& Quast, G. R. 1985, Rev. Mex. Astron. Astrofis., 10, 329

Van Leeuwen, F., Alphenaar, P., \& Meys, J. J. M. 1987, A\&AS, 67,483

Vilhu, O. 1984, A\&A, 133, 117

Vogt, S. S. 1975, ApJ, 199, 418

Ziegler, K., Shirley, M., Francam, B., Florence, W., \& Hall, D. S. 1984, IBVS, 2619 